\title{
Expanded horizons for generating and exploring optical angular momentum in vortex structures
}

\author{
David L. Andrews, Matt M. Coles, Mathew D. Williams and David S. Bradshaw \\ School of Chemistry, University of East Anglia, Norwich Research Park, Norwich NR4 7TJ, U. K.
}

\begin{abstract}
Spin provides for a well-known extension to the information capacity of nanometer-scale electronic devices. Spin transfer can be effected with high fidelity between quantum dots, this type of emission being primarily associated with emission dipoles. However, in seeking to extend the more common spectroscopic connection of dipole transitions with orbital angular momentum, it has been shown impossible to securely transmit information on any other multipolar basis - partly because point detectors are confined to polarization measurement. Standard polarization methods in optics provide for only two independent degrees of freedom, such as the circular states of opposing handedness associated with photon spin. Complex light beams with structured wave-fronts or vector polarization do, however, offer a basis for additional degrees of freedom, enabling individual photons to convey far more information content. A familiar example is afforded by Laguerre-Gaussian modes, whose helically twisted wave-front and vortex fields are associated with orbital angular momentum. Each individual photon in such a beam has been shown to carry the entire spatial helical-mode information, supporting an experimental basis for sorting beams of different angular momentum content. One very recent development is a scheme for such optical vortices to be directly generated through electronic relaxation processes in structured molecular chromophore arrays.
\end{abstract}

Keywords: nanophotonics, optical vortex; optical angular momentum; quantum dots; surface plasmon; multipole optical emission, nanoscale materials, quantum electrodynamics

\section{INTRODUCTION}

It is widely appreciated that spin offers a useful extension to the information capacity of nanometer-scale electronic devices, and that it is possible for spin transfer to effect high fidelity communication between component units such as quantum dots [1-5]. The emission from these components is largely associated with emission dipoles, and to secure emission of intricate multipolar form might suggest the use of lower symmetry, molecular systems; however, it emerges that such emission cannot convey unambiguous information on multipolar content [6,7]. In the field of optics, where standard polarization methods provide for two independent degrees of freedom to be communicated by photons, circular states of opposing handedness associated with photon spin are the most obvious candidates, although any two states corresponding to diametrically opposite positions on the Poincaré sphere can provide an equally valid basis. Nonetheless, it has been shown that a whole family of optical chirality measures represents no other degrees of freedom than that of spin angular momentum [8]. Various forms of complex light with structured wave-fronts or vector (e.g. radial or azimuthal) polarization do, however, provide for the long-sought additional degrees of freedom, enabling individual photons to convey a far greater information content than was previously thought possible. There is particular interest in Laguerre-Gaussian modes, whose helically twisted wave-front is associated with orbital angular momentum, in addition to any spin due to polarization. Experiments demonstrate that single photons carry the entire spatial helicalmode information, supporting an experimental basis for sorting beams of different angular momentum content [9].

Spintronics VI, edited by Henri-Jean Drouhin, Jean-Eric Wegrowe,

Manijeh Razeghi, Proc. of SPIE Vol. 8813, 88130Y - @ 2013 SPIE

CCC code: $0277-786 X / 13 / \$ 18 \cdot$ doi: $10.1117 / 12.2025141$

Proc. of SPIE Vol. $881388130 Y-1$ 


\section{QUANTUM DOT SPIN TRANSFER}

For the electron, the familiar spin-up/spin-down state designation signifies and provides for a two-level basis in quantum theory, affording a potential basis for the transmission of qubit information [6,7]. The underlying principle is a separation of spin $\mathbf{S}$ and orbital angular momentum $\mathbf{L}$ which owes its origin to the commutation of the Hamiltonian and $\mathbf{L}^{2}$ operators - itself a corollary of the radial symmetry in atoms (although a relativistic formulation makes it apparent that spin-orbit coupling undermines complete fidelity in such a separation). To see how this principle applies in a simple solid state context it is instructive to consider a quantum dot (QD) system, which exemplifies the association of electron spin information transfer with circularly polarized light. The nature of the electronic energy level structure in quantum dots is responsible for their distinctive and widely deployed optical characteristics - in particular the discrete, sizetunable, and intense character of quasi two-level exciton transitions. Such transitions are usually dipolar in nature. Particular interest is drawn by the fine structure that underlies some of these transitions - for example in the $1 \mathrm{~S}_{3 / 2}-1 \mathrm{~S}_{\mathrm{e}}$ transition observed in CdSe quantum dots [12]. The selection rules for excitation by circularly polarized light allow for exciton states of specific spin to be optically oriented, and in consequence optically bright exciton states can be distinguished using circularly polarizations - provided the QDs are suitably oriented.

Electronic energy can migrate within a system of quantum dots by the process of resonance energy transfer (RET) [13]. It has been established by an analysis based on QED (quantum electrodynamics) that the spin state of an exciton can be transmitted or reversed by RET between suitably oriented quantum dots [3]. This analysis identifies contributions to the quantum amplitude corresponding to coupling by left- and right-circularly polarized virtual photons. The plots shown in Fig. 1 exhibit the result of rotating one quantum dot relative to the other: when the two QDs have parallel transition moments, the exciton spin orientation is transmitted with complete fidelity from one QD to the other; in contrast, an antiparallel configuration provides for a reversal of the spin. This signifies, for example, that electronic energy migration along a QD column oriented in a common direction will faithfully retain information on spin orientation from the initial donor particle. In the long-range asymptote, it is clear that angular momentum should indeed be conserved about the propagation direction of the photon, coinciding with the displacement vector of adjacent quantum dots. However, the QED calculation additionally reveals the less obvious finding that exactly the same feature operates in the near-zone region, even though the coupling cannot in this case be ascribed to real photon propagation. Energy migration along a column of quantum dots with a common orientation preserves spin information; the observation of spin reversal between alternately inverted quantum dots is another manifestation of the same principle.
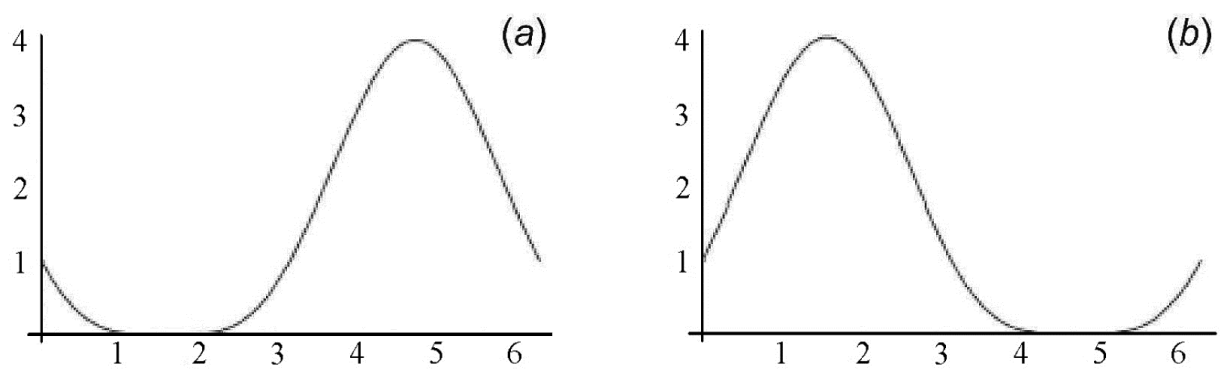

Figure 1. Variation in the rate of quantum dot energy transfer, over one complete cycle of the mutual orientation: $(a)$ spin antiparallel; (b) spin parallel. 


\section{MULTIPOLAR EMISSION AND DETECTION}

The coupling between quantum dots represents a principle that invites extension beyond the two-state case where excitons can have a simple spin up or spin-down designation and the transitions all have dipolar character. Taking a more general perspective, multipolar transitions might appear to offer an opportunity to broaden this basis, without involving electron spin. However, it has been shown that the transfer of an associated angular momentum does not in general occur with high fidelity between any individual energy donor and acceptor [6]. The underlying reason is that multipolar decay does not itself produce photons measurably imprinted with the corresponding angular momentum - the character of the emission is only registered statistically, in the spatial distribution of the emerging radiation. Any individually positioned detector is confined to polarization measurement.

For molecules, or atoms placed in anisotropic environments, an additional tier of complexity arises. In such systems full rotational symmetry is compromised, and accordingly most electronic states cannot be uniquely characterized in terms of angular momentum, nor can each electronic transition be unambiguously associated with a specific angular momentum change; a given transition will commonly be allowed by more than one form of multipolar coupling. Here, it proves impossible to unambiguously determine, with any conventional detector, the multipolar character of any decay transition associated with its generation [6,7].

As in the case of the coupled quantum dots, the analysis here begins with a general expression for the overall emission and detection process. The focus is on the electrodynamic coupling between two transition electric multipoles E $m$ and En, the former signifying the decay of a source particle $A$ and the latter, excitation of the detector $B$ (using the shorthand $\mathrm{E} n$ to denote an electric multipolar interaction of order $n$, E2 denoting electric quadrupole, for example). The quantum amplitude for the coupling result can be generally cast as;

$$
M_{f i}^{\mathrm{E} m-\mathrm{E} n}=E_{A ; a_{1} \ldots a_{m}}^{(m)} V_{a_{1} \ldots a_{m} b_{1} \ldots b_{n}}(k, \boldsymbol{R}) E_{B ; b_{1} \ldots b_{n}}^{(n)}
$$

using the convention of implied summation over repeated Cartesian (lower-case subscript) indices. In equation (1), $\boldsymbol{E}_{\xi}^{(m)}$ is the transition multipole tensor of order $m$ for particle $\zeta$, and the following is a series expansion for the coupling tensor that engages $\mathrm{E} m$ source emission at A with $\mathrm{E} n$ detection at B;

$$
V_{a_{1} \ldots a_{m} b_{1} \ldots b_{n}}(k, \boldsymbol{R})=\left(4 \pi \varepsilon_{0}\right)^{-1} k^{m+n+1} e^{i k R} \sum_{p=1}^{m+n+1}(k R)^{-p} W_{a_{1} \ldots a_{m} b_{1} \ldots b_{n}}^{(p-1)}(\hat{\boldsymbol{R}})
$$

Here, $R$ is the distance of $B$ from $A, k$ is the optical wave-number for the conveyed energy, and the entire tensor and angular momentum character is distributed between individual $\boldsymbol{W}^{(p)}(\hat{\boldsymbol{R}})$ tensors, in each of which the superscript identifies the associated inverse power of $k R$.

Focusing on the E2-E1 case for example, where the emitter undergoes an electric quadrupole decay and the detector has the standard dipole form, i.e. $m=2, n=1$, the index $p$ on the summation in equation (2) runs from 1 to $2+1+1$, signifying that the coupling has terms running from $R^{-1}$ to $R^{-4}$. Thus it emerges that the coupling accommodates the following nonzero angular momentum contributions: 


$$
p=1\left(R^{-1}\right): j=1,3 ; \quad p=2\left(R^{-2}\right): j=1,3 ; \quad p=3\left(R^{-3}\right): j=3 ; \quad p=4\left(R^{-4}\right): j=3 .
$$

Here, the short-range contribution $(p=4)$ with $j=3$ is consistent with a coupling that can accommodate an exchange of three units of angular momentum - two engaging the source, and one the detector. At longer distances, lower inverse powers of $R$ become prominent and the coupling permits values of both $j=1$ and $j=3$ for the conferred orbital angular momentum.

In general, in the wave-zone $\left(k R>>1\right.$ ), the term with the lowest inverse power of $R$ (always $R^{-1}$, associated with the inverse square law for the rate) dominates $V_{a_{1} \ldots a_{m} b_{1} \ldots b_{n}}(k, \boldsymbol{R})$, and the resulting $F_{b_{1} \ldots b_{n}}(k, \boldsymbol{R})$ entails a spectrum of integer weights in the range $(0 \leq j \leq n)$. Subject to the exclusion of alternate weights on symmetry grounds, each allowed weight in the generated field can deliver a corresponding quantity of angular momentum. The multipolar electronic excitation of the detector necessarily engages only the irreducible component of the field that confers the requisite angular momentum; the implication is that the nature of the detector itself imposes an upper bound. This discovery is perfectly consistent with the angular quantum uncertainty principle [14,15]: the removal of a detector from the vicinity of the source produces a decreasing angular uncertainty in photon propagation direction. This is manifest in an increasing range of possible integer values for the angular momentum of the detected light.

\section{THE POLARIZATION BASIS IN OPTICS}

In the field of optics, several different strands of research have led to a renewed optimism over the possibility of encoding and optically transmitting higher dimensionality information [14-21]. One feature that has aroused considerable recent interest is the rediscovered quantitation of 'optical chirality' and its associated flux for classical and quantum optical fields [22-25]. These conserved quantities were originally extracted from Maxwell's equations with an application of Noether's Theorem, with the earliest reporter being surprisingly dismissive of the results, surmising that they could have no physical significance [26]. Appealing to this earlier work, there have been more recent suggestions that there might exist 'superchiral' regions of some electromagnetic fields, exhibiting circular differential effects higher than that of pure circularly polarized light [27-30]. However, such suggestions have met with opposition. Both classical and quantum calculations of these measures clearly indicate that optical chirality has maximum (or minimum) values for fields comprising solely left- or right- handed light, and therefore the term 'superchiral' proves redundant [31].

To address the connection between the measures of optical chirality and the well-known optical angular momentum, we divide the latter into spin and orbital components [32,33]:

$$
\mathbf{J}=\varepsilon_{0} \int d^{3} \mathbf{r}[\mathbf{r} \times(\mathbf{E} \times \mathbf{B})]
$$

where $\varepsilon_{0}$ is the vacuum permittivity, $\mathbf{E}$ and $\mathbf{B}$ are the electric and magnetic fields respectively; $\mathbf{r}$ is the position vector and the $\mathbf{r}$ dependence of $\mathbf{E}(\mathbf{r})$ and $\mathbf{B}(\mathbf{r})$ is implicit. The optical angular momentum can be constructed as the sum of the orbital and spin components,

$$
\begin{aligned}
& \mathbf{L}=\varepsilon_{0} \int d^{3} \mathbf{r} E_{i}(\mathbf{r} \times \nabla) \cdot A_{i}, \\
& \mathbf{S}=\varepsilon_{0} \int d^{3} \mathbf{r}(\mathbf{E} \times \mathbf{A}),
\end{aligned}
$$


respectively. Here A represents the electromagnetic vector potential and the Einstein summation convention is used for repeated indices. It is tempting to identify, in the above separation of spin and orbital angular momenta (OAM) for light, a principle similar to the counterpart separation of spin and orbital angular momenta for the electron - but again there is a proviso, namely that the paraxial approximation can be applied. Indeed, it is entirely wrong to assume any association between electronic and optical forms of orbital angular momentum.

By invoking the electric-magnetic democracy the spin component is delivered in a symmetric form:

$$
\mathbf{S}=\frac{\varepsilon_{0}}{2} \int d^{3} \mathbf{r}(\mathbf{E} \times \mathbf{A}+\mathbf{B} \times \mathbf{C}),
$$

where $\mathbf{C}$ is a second (pseudo)vector potential, with $\mathbf{E}=-\nabla \times \mathbf{C}$. By introducing the optical helicity as the projection of the spin onto the propagation direction, we obtain:

$$
h=\frac{\varepsilon_{0}}{2} \int d^{3} \mathbf{r}(\mathbf{A} \cdot \mathbf{B}-\mathbf{E} \cdot \mathbf{C}) .
$$

Eq. (6) and (7) can be written in index-contracted form as $\partial^{\mu} S_{\mu}=0$; the optical helicity and its flux, spin, together represent components of a 4-vector $(c h, \mathbf{S})$ in Minkowski space, where $c$ is the speed of light. It has been shown by Cameron et al. that replacing the vector potentials and fields by their curls (or time-derivatives) in Eq. (6) and (7) deliver the optical chirality flux and the optical chirality respectively [34]:

$$
\begin{gathered}
\boldsymbol{\varphi}=\frac{\varepsilon_{0} c^{2}}{2} \int d^{3} \mathbf{r}(\mathbf{E} \times(\nabla \times \mathbf{B})-\mathbf{B} \times(\nabla \times \mathbf{E})) \\
\chi=\int d^{3} \mathbf{r}\left(\frac{\varepsilon_{0}}{2} \mathbf{E} \cdot \nabla \times \mathbf{E}+\frac{1}{2 \mu_{0}} \mathbf{B} \cdot \nabla \times \mathbf{B}\right),
\end{gathered}
$$

where $\mu_{0}$ is the magnetic permeability. Moreover, repeated application of this prescription delivers an infinite hierarchy of helicity-type and spin-type measures.

In the framework of quantum optics, the fields and potentials are promoted to Hilbert space operators; the electromagnetic vector potential for a plane wave is given by [35]:

$$
\mathbf{A}=\sum_{\mathbf{k}, \eta}\left(\frac{\hbar}{2 \varepsilon_{0} c k V}\right)^{\frac{1}{2}}\left\{\mathbf{e}^{(\eta)}(\mathbf{k}) a^{(\eta)}(\mathbf{k}) e^{i(\mathbf{k} \cdot \mathbf{r})}+\overline{\mathbf{e}}^{(\eta)}(\mathbf{k}) a^{\dagger(\eta)}(\mathbf{k}) e^{-i(\mathbf{k} \cdot \mathbf{r})}\right\},
$$

where $\hbar$ is the reduced Plank constant and $V$ the quantization volume, while $a^{(\eta)}(\mathbf{k})$ is the photon annihilation operator for a mode with polarization $\eta$ and wave-vector $\mathbf{k}$. The electric and magnetic field operators can be derived from the vector potential by $\mathbf{E}=-\partial \mathbf{A} / \partial t$ and $\mathbf{B}=\nabla \times \mathbf{A}$. The polarization vector $\mathbf{e}^{(\eta)}(\mathbf{k})$ is usually summed over a convenient basis set, e.g. linearly or circularly polarized light; however, we can introduce a more general pair of basis vectors. Any pair of polarization vectors that sit diametrically opposed on the Poincare sphere, Fig. 2, are orthogonal in the sense that $\mathbf{e}^{(n)}(\mathbf{k}) \cdot \mathbf{e}^{(m)}(\mathbf{k})=\delta_{n m}$. Introduction of a general polarization vector $\mathbf{e}_{1}=\sin \theta \hat{\mathbf{i}}+e^{i \phi} \cos \theta \hat{\mathbf{j}}$, thus allows the corresponding 


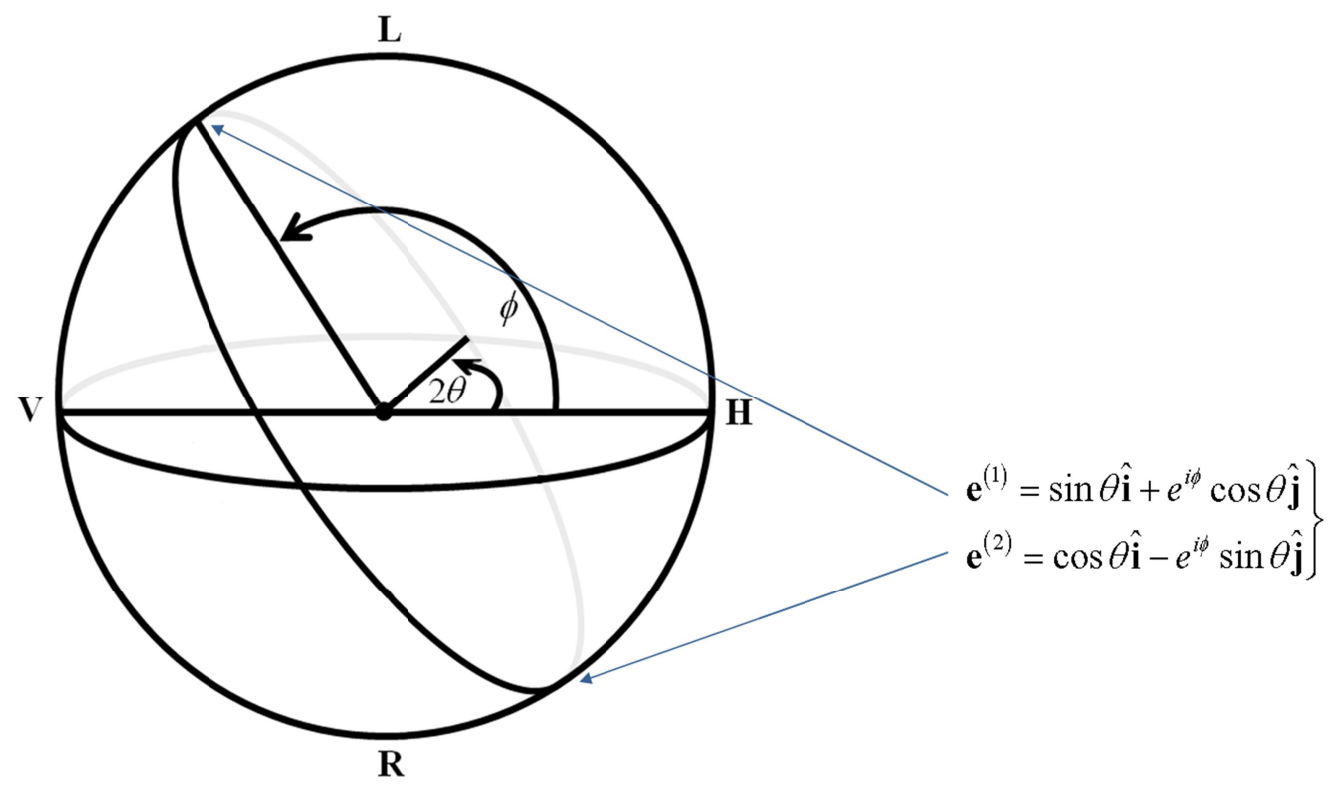

Figure 2. The Poincaré sphere and a depiction of two diametrically opposing basis states. Here, the generalized expressions for polarization states are orthogonal in the sense that $\mathbf{e}^{(n)} \cdot \overline{\mathbf{e}}^{(m)}=\delta_{n m}$.

opposing basis vector to be delivered as $\mathbf{e}_{2}=\cos \theta \hat{\mathbf{i}}-e^{i \phi} \sin \theta \hat{\mathbf{j}}$. Importantly, all helicity-type and spin-type measures, including the optical chirality, are delivered, in a quantum optical framework, as dependent upon:

$$
\sim \sin (2 \theta) \sin (\varphi) \sum_{\mathbf{k}}\left\{\hat{N}^{(1)}(\mathbf{k})-\hat{N}^{(2)}(\mathbf{k})\right\}
$$

where $\hat{N}^{(\eta)}(\mathbf{k})=a^{\dagger(\eta)}(\mathbf{k}) a^{(\eta)}(\mathbf{k})$ is the number operator for each mode [31]. Notably, this dependence on number operators of opposing helicity delivers maximal values for purely left- or right- handed light, and vanishes when the light is linearly polarized. In conclusion, the various measures of helicity and chirality have the same basis sets. These higher-order spins do not, then, indicate an improved information capacity for a single photon, over that contained in the spin angular momentum.

\section{OPTICAL VORTICES AND ORBITAL ANGULAR MOMENTUM}

In the past twenty-five years the understanding of optical angular momentum has undergone a huge development, stimulated by the realization that it is possible to optically engineer structured beams of light that can convey orbital angular momentum, as a result of phase singularities and associated vortex structures in the beam wave-front [36-38]. Distinct from the unit spin angular momentum connected with circularly polarized light, suitably engineered 'twisted beams' can convey significant integer multiples of the fundamental unit of angular momentum, $\hbar$, per photon $[39,40]$. Most of the research in this area has focused on Laguerre-Gaussian (LG) modes of light. Here, we deploy quantum expansions for the vector potential of an LG mode as a test case for vortex beams. In the paraxial approximation, the magnetic and transverse electric field vectors can again be determined from the vector potential, now given by 


$$
\mathbf{A}=\sum_{\mathbf{k}, \eta, l, p}\left(\frac{\hbar}{2 \varepsilon_{0} c k V}\right)^{\frac{1}{2}}\left\{\mathbf{e}_{l, p}{ }^{(\eta)}(\mathbf{k}) a^{(\eta)}(\mathbf{k}) f_{l, p}(r) e^{i k z-i l \varphi}+\overline{\mathbf{e}}_{l, p}{ }^{(\eta)}(\mathbf{k}) a^{\dagger(\eta)}(\mathbf{k}) f_{l, p}(r) e^{-i k z+i l \varphi}\right\}
$$

where $f_{l, p}(r)$ represents the radial distribution of the LG mode with radial number $p$ and azimuthal index $l$. When applied to a field of mode $(\mathbf{k}, \lambda, l, p)$ the operator becomes:

$$
L=\hbar \sum_{\mathbf{k}, l, p} l \hat{\mathbf{k}}\left[\hat{N}_{l p}^{(1)}(\mathbf{k})+\hat{N}_{l p}^{(2)}(\mathbf{k})\right] .
$$

In the case of plane waves, the analysis of orbital angular momentum gives a vanishing result. However, for light carrying OAM, the measure takes on unbounded integer values. Separation into spin and orbital angular momentum in the paraxial approximation is thus equivalent to dividing the optical angular momentum into parts that respectively depend on the difference, and sum, of number operators.

There have been many propositions for methods that could deploy individual photons to encode information on beam structure - particularly topological charge (which, for LG beams, equates to the azimuthal index). Boyd et al. have shown that is possible to experimentally separate beams with different $l$ values [41], while others have shown that modal information encoded in single-photon states can be resolved by beam tomography [42]. The synthesis detection and visualization of optical vortex modes are key areas of current development [43-45]. Such studies have stimulated the conception of numerous schemes for quantum communication and data handling applications, but the issue of total information content that can be contained in these degrees of freedom is still unresolved. In particular, any such scheme has to be reconciled with the angle-angular momentum uncertainty principle for photons [46]. Surprisingly this principle, of paramount relevance to low-number states, has seemingly received very little consideration in the literature to date.

Mention should also be made of the growing area of surface plasmon optical vortices [47], which is one of the more recently discovered phenomena to emerge from the study of light conveying OAM. As is well known, surface plasmons are forms of electromagnetic excitation in which the fluctuational motions of conduction electrons, generally on the surfaces of noble metals, are entangled with light at optical frequencies. These plasmons are widely associated with strongly enhanced optical properties. In principle it is straightforward to generate a surface plasmon optical vortex (SPOV) using twisted light. For example, an LG beam impinging on a thin metallic layer will fulfill the basic conditions. When such a film is adsorbed on the surface of a transparent substrate, the beam can engage with the conduction electrons at the surface through the process of total internal reflection, imparting a circulation of charge. The light field in the vacuum region is evanescent, decaying with distance away from the film surface, but it retains the phase singularity of the original light beam and also the associated OAM. To offset a linear momentum along the surface, in the reflection plane, it is expedient to use a counter-propagating beam geometry as shown in Fig. $3(a)$, using beams with opposite sign $l$ values. Very recently an alternative method that helps overcome the need for precise beam localization has been demonstrated by $\mathrm{Ku}$ et al., based on circularly polarized plane-wave excitation of a plasmonic Archimedes spiral, with the SPOV formation deterministically tailored by the geometrical design [48]. Shen et al. have also demonstrated an effective method for measuring the OAM and intensity distribution of an SPOV using near-field scanning optical microscopy [49].

An atom or atoms trapped near the surface by a local SPOV field will be subject to forces associated with a potential energy surface in the form of an elliptical valley, its height defined by the intensity distribution. A particularly 
interesting is where the two counter-propagating vortex beams have a slightly different optical frequency [50]. Here, the pattern of interference between the surface optical vortices produces a rotation at the difference frequency. Particles trapped in this local field will therefore experience an accelerating circular motion, with the radial confinement leading to vibrational motion in a radial direction and thus resulting in an overall zigzag trajectory, as shown in Fig. 3(b). Here, applications can be envisaged in atom lithography, alongside the further development of optical tweezers and optical wrench (spanner) techniques.

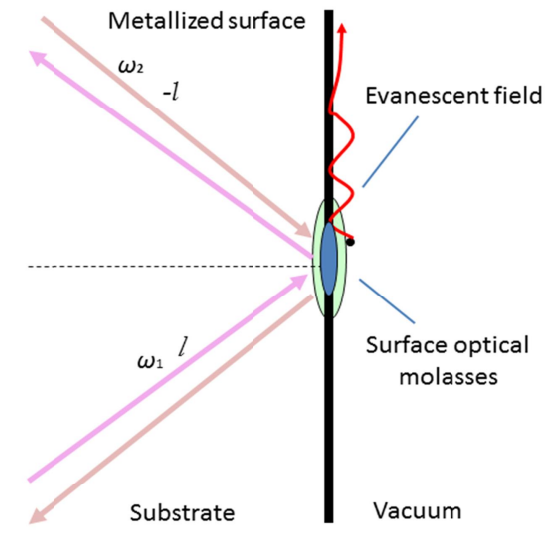

(a)

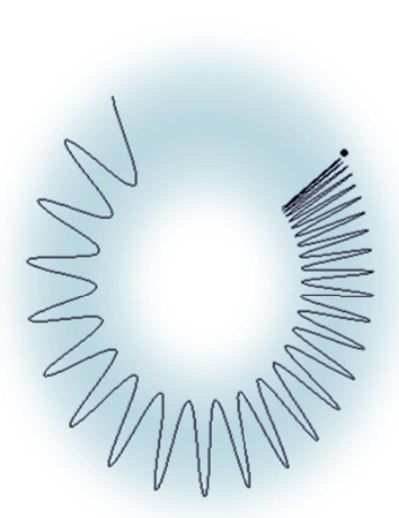

(b)

Figure 3. (a) The generation of a surface plasmon optical vortex at a metallized surface. (b) Counter-propagating beams of similar optical frequency but opposite topological charge generate a potential energy surface of elliptical form, in which a trapped atom exhibits an accelerating zigzag motion around the singularity.

\section{OPTICAL VORTEX GENERATION}

The generation of light endowed with orbital angular momentum is usually accomplished by passing a plane-wave beam through an optical element, such as a spatial light modulator [51,52] or a spiral phase plate [53]. The conversion of light through q-plates additionally addresses the issue of coupling the optical spin and orbital degrees of freedom [54,55]. However, it has not been considered possible, until recently [56], to directly generate optical vortex light with a predetermined azimuthal index. Furthermore, it is known that in the interaction of molecules with optical vortex light, OAM exchange only occurs between the radiation and the centre-of-mass motion, when considering electric dipole transitions [57].

A conventional atomic or molecular source does not satisfy the specific symmetry-based criteria required for the emission of photons conveying OAM. However, a recent theoretical study suggests that the demanding conditions can be satisfied by suitably tailored arrays of chromophore nanoantennas [58]. The requirement is for a number of chromophores, $n$, with non-parallel transition dipoles, to be placed at each vertex of a regular $n$-polygon with $n$-fold rotational symmetry about the centre of the array. Such an array will have Schoenflies point group designation $C_{n}$ or $C_{n h}$, depending on whether the array has mirror symmetry in the plane containing the centre-of-charge of each emitter. 
Molecular systems corresponding to such point groups have electronic state pairs in the form of degenerate excitons, each of which has a wavefunction with exactly the phase progression required for the production of an OAM photon. In such cases, each component of a degenerate pair decays to produce radiation with an opposite sense of helical twist. It is possible to secure a specific handedness of emission by causing a small shift in the symmetry of the array, by either embedding the emitters in an anisotropic substrate, or by deformation of the array. Without this symmetry breaking, such arrays produce photons in superposition states, potentially lending insight into the study of highly entangled quantum systems.

For an array consisting of $n$ optical centers, with the above characteristics, the point group demands that there exist $L(n-$ $1) / 2\rfloor$ (signifying the largest integer not greater than $(n-1) / 2$ ) doubly degenerate excitons, each component of which can decay to produce a photon with maximum topological charge also given by $\lfloor(n-1) / 2\rfloor$. [There is, in addition, a nondegenerate state of circular symmetry, forbidding optical vortex emission.] As these degenerate states are all separate in energy, they could in principle be selectively excited - and so, through their decay, produce any of the allowed optical vortex modes. With suitable attention to a convenient pump mechanism, it might be supposed that large numbers of phase-matched nanoarray units (for example, stacked within the cavities of a porous silicate) might indeed provide the basis for an optical vortex laser.

\section{CONCLUSION}

The concepts and phenomena associated with spin and orbital angular momentum, in atoms and molecules and in light, provide a rich area of science - and one in which a stream of new discoveries is coming to the fore. Some of the most interesting are phenomena in which interactions within these areas are associated with chirality, both in its manifestations and measurements. Against the backdrop of well-established techniques such as circular dichroism, for example, there are now reports of films of chiral molecules selectively transmitting electrons with a certain spin, and so acting as a spin filter for photoelectrons ejected from a gold surface [59]. As new methods for the production of optical vortex light continue to emerge, the scope for exploiting light with orbital angular momentum, in particular, appears likely to be a source of much further development in this field, offering scope for applications ranging from quantum informatics and nanoparticle manipulation through to nanolithography.

\section{ACKNOWLEDGMENT}

The authors gratefully acknowledge collaborations with other authors whose work is prominently featured here, especially Greg Scholes (University of Toronto), Mohamed Babiker (University of York) and Vassilis Lembessis (King Saud University). Research in the QED group at the University of East Anglia is funded by the Leverhulme Trust and the Engineering and Physical Sciences Research Council.

\section{REFERENCES}

[1] Ouyang, M. and Awschalom, D. D., "Coherent spin transfer between molecularly bridged quantum dots," Science 301, 1074-1078 (2003). 
[2] Rippard, W. H., Pufall, M. R., Kaka, S., Silva, T. J., Russek, S. E. and Katine, J. A., "Injection locking and phase control of spin transfer nano-oscillators," Phys. Rev. Lett. 95, 067203 (2005).

[3] Scholes, G. D. and Andrews, D. L., "Resonance energy transfer and quantum dots," Phys. Rev. B 72, 125331 (2005).

[4] Scholes, G. D., Andrews, D. L., Huxter, V. M., Kim, J. and Wong, C. Y., "Transmission of quantum dot exciton spin states via resonance energy transfer," Proc. SPIE 5929, 59290H (2005).

[5] Zeng, Z., Finocchio, G., Zhang, B., Amiri, P. K., Katine, J. A., Krivorotov, I. N., Huai, Y., Langer, J., Azzerboni, B., Wang, K. L. and Jiang, H., "Ultralow-current-density and bias-field-free spin-transfer nano-oscillator," Sci. Rep. 3, 1426 (2013).

[6] Andrews, D. L., “Optical angular momentum: Multipole transitions and photonics," Phys. Rev. A 81, 033825 (2010).

[7] Andrews, D. L., "On the conveyance of angular momentum in electronic energy transfer," Phys. Chem. Chem. Phys. 12, 7409-7417 (2010).

[8] Coles, M. M. and Andrews, D. L., "Photonic measures of helicity: optical vortices and circularly polarized reflection,” Opt. Lett. 38, 869-871 (2013).

[9] Fickler, R., Krenn, M., Lapkiewicz, R., Ramelow, S. and Zeilinger, A., "Real-time imaging of quantum entanglement," Sci. Rep. 3, 1914 (2013).

[10] Vrijen, R., Yablonovitch, E., Wang, K., Jiang, H. W., Balandin, A., Roychowdhury, V., Mor, T. and DiVincenzo, D., "Electron-spin-resonance transistors for quantum computing in silicon-germanium heterostructures," Phys. Rev. A 62, 012306 (2000).

[11] Childress, L., Gurudev Dutt, M. V., Taylor, J. M., Zibrov, A. S., Jelezko, F., Wrachtrup, J., Hemmer, P. R. and Lukin, M. D., "Coherent dynamics of coupled electron and nuclear spin qubits in diamond," Science 314, 281285 (2006).

[12] Wang, H., de Mello Donegá, C., Meijerink, A. and Glasbeek, M., "Ultrafast exciton dynamics in CdSe quantum dots studied from bleaching recovery and fluorescence transients," J. Phys. Chem. B 110, 733-737 (2005).

[13] Bradshaw, D. S. and Andrews, D. L., "All-optical switching between quantum dot nanoarrays," Superlatt. Microstruct. 47, 308-313 (2010).

[14] Pegg, D. T., Vaccaro, J. A. and Barnett, S. M., "Quantum-optical phase and canonical conjugation,” J. Mod. Opt. 37, 1703-1710 (1990).

[15] Leach, J., Jack, B., Romero, J., Jha, A. K., Yao, A. M., Franke-Arnold, S., Ireland, D. G., Boyd, R. W., Barnett, S. M. and Padgett, M. J., "Quantum correlations in optical angle-orbital angular momentum variables," Science 329, 662-665 (2010).

[16] Hasegawa, A., "Theory of information transfer in optical fibers: A tutorial review," Opt. Fiber Technol. 10, 150170 (2004). 
[17] Liu, C., Dutton, Z., Behroozi, C. H. and Hau, L. V., "Observation of coherent optical information storage in an atomic medium using halted light pulses," Nature 409, 490-493 (2001).

[18] Gibson, G., Courtial, J., Padgett, M., Vasnetsov, M., Pas'ko, V., Barnett, S. and Franke-Arnold, S., "Free-space information transfer using light beams carrying orbital angular momentum," Opt. Express 12, 5448-5456 (2004).

[19] Jia, P., Yang, Y., Min, C. J., Fang, H. and Yuan, X.-C., "Sidelobe-modulated optical vortices for free-space communication," Opt. Lett. 38, 588-590 (2013).

[20] Veissier, L., Nicolas, A., Giner, L., Maxein, D., Sheremet, A. S., Giacobino, E. and Laurat, J., "Reversible optical memory for twisted photons," Opt. Lett. 38, $712-714$ (2013).

[21] Wang, J., Yang, J.-Y., Fazal, I. M., Ahmed, N., Yan, Y., Huang, H., Ren, Y., Yue, Y., Dolinar, S., Tur, M. and Willner, A. E., "Terabit free-space data transmission employing orbital angular momentum multiplexing," Nat. Photon. 6, 488-496 (2012).

[22] Coles, M. M. and Andrews, D. L., "Chirality and angular momentum in optical radiation," Phys. Rev. A 85, 063810 (2012).

[23] Bliokh, K. Y. and Nori, F., “Characterizing optical chirality,” Phys. Rev. A 83, 021803 (2011).

[24] Tang, Y. and Cohen, A. E., "Optical chirality and its interaction with matter," Phys. Rev. Lett. 104, 163901 (2010).

[25] Fushchich, W. I. and Nikitin, A. G., "The complete sets of conservation laws for the electromagnetic field," J. Phys. A 25, L231 (1992).

[26] Lipkin, D. M., "Existence of a new conservation law in electromagnetic theory," J. Math. Phys. 5, 696-700 (1964).

[27] Tang, Y. and Cohen, A. E., "Enhanced enantioselectivity in excitation of chiral molecules by superchiral light," Science 332, 333-336 (2011).

[28] Yang, N. and Cohen, A. E., "Local geometry of electromagnetic fields and its role in molecular multipole transitions," J. Phys. Chem. B 115, 5304-5311 (2011).

[29] Hendry, E., Carpy, T., Johnston, J., Popland, M., Mikhaylovskiy, R. V., Lapthorn, A. J., Kelly, S. M., Barron, L. D., Gadegaard, N. and Kadodwala, M., "Ultrasensitive detection and characterization of biomolecules using superchiral fields," Nat. Nano. 5, 783-787 (2010).

[30] Hendry, E., Mikhaylovskiy, R. V., Barron, L. D., Kadodwala, M. and Davis, T. J., "Chiral electromagnetic fields generated by arrays of nanoslits," Nano Lett. 12, 3640-3644 (2012).

[31] Andrews, D. L. and Coles, M. M., "Measures of chirality and angular momentum in the electromagnetic field," Opt. Lett. 37, 3009-3011 (2012).

[32] Allen, L., Barnett, S. M. and Padgett, M. J., [Optical Angular Momentum], Taylor \& Francis Group, Bristol (2003).

[33] Andrews, D. L. and Babiker, M., [The Angular Momentum of Light], Cambridge University Press, Cambridge, UK (2013). 
[34] Barnett, S. M., Cameron, R. P. and Yao, A. M., "Duplex symmetry and its relation to the conservation of optical helicity," Phys. Rev. A 86, 013845 (2012).

[35] Craig, D. P. and Thirunamachandran, T., [Molecular Quantum Electrodynamics: An Introduction to RadiationMolecule Interactions], Dover Publications, Mineola, NY (1998).

[36] Nye, J. F. and Berry, M. V., "Dislocations in wave trains," Proc. R. Soc. A 336, 165-190 (1974).

[37] Basistiy, I. V., Bazhenov, V. Y., Soskin, M. S. and Vasnetsov, M. V., "Optics of light beams with screw dislocations," Opt. Commun. 103, 422-428 (1993).

[38] Franke-Arnold, S., Allen, L. and Padgett, M., “Advances in optical angular momentum,” Laser \& Photon. Rev. 2, 299-313 (2008).

[39] Allen, L., Beijersbergen, M. W., Spreeuw, R. J. C. and Woerdman, J. P., "Orbital angular momentum of light and the transformation of Laguerre-Gaussian laser modes," Phys. Rev. A 45, 8185-8189 (1992).

[40] Andrews, D. L., [Structured Light and its Applications: An Introduction to Phase-Structured Beams and Nanoscale Optical Forces], Academic, Amsterdam, Boston (2008).

[41] O'Sullivan, M. N., Mirhosseini, M., Malik, M. and Boyd, R. W., "Near-perfect sorting of orbital angular momentum and angular position states of light," Opt. Express 20, 24444-24449 (2012).

[42] Barreiro, J. T., Wei, T.-C. and Kwiat, P. G., "Remote preparation of single-photon 'hybrid' entangled and vectorpolarization states," Phys. Rev. Lett. 105, 030407 (2010).

[43] Genevet, P., Lin, J., Kats, M. A. and Capasso, F., "Holographic detection of the orbital angular momentum of light with plasmonic photodiodes," Nat. Commun. 3, 1278 (2012).

[44] Ku, C.-D., Huang, W.-L., Huang, J.-S. and Huang, C.-B., "Deterministic synthesis of optical vortices in tailored plasmonic archimedes spiral,” IEEE Photon. J. 5, 4800409-4800409 (2013).

[45] Shen, Z., Hu, Z. J., Yuan, G. H., Min, C. J., Fang, H. and Yuan, X. C., "Visualizing orbital angular momentum of plasmonic vortices," Opt. Lett. 37, 4627-4629 (2012).

[46] Jack, B., Aursand, P., Franke-Arnold, S., Ireland, D. G., Leach, J., Barnett, S. M. and Padgett, M. J., "Demonstration of the angular uncertainty principle for single photons," J. Opt. 13, 064017 (2011).

[47] Lembessis, V. E., Babiker, M. and Andrews, D. L., "Surface optical vortices," Phys. Rev. A 79, 011806(R) (2009).

[48] Ku, C. D., Huang, W. L., Huang, J. S. and Huang, C. B., "Deterministic Synthesis of Optical Vortices in Tailored Plasmonic Archimedes Spiral,” IEEE Photonics J. 5, 4800409 (2013).

[49] Shen, Z., Hu, Z. J., Yuan, G. H., Min, C. J., Fang, H. and \& Yuan, X. C., "Visualizing orbital angular momentum of plasmonic vortices," Opt. Lett. 37, 4627-4629 (2012).

[50] Andrews, D. L., Babiker, M., Lembessis, V. E. and Al-Awfi, S., "Surface plasmons with phase singularities and their effects on matter," Phys. Status Solidi RRL, 4, 241-243 (2010). 
[51] Ostrovsky, A., Rickenstorff-Parrao, C. and Arrizón, V., "Generation of the "perfect" optical vortex using a liquid-crystal spatial light modulator," Opt. Lett. 38, 534-536 (2013).

[52] Heckenberg, N., McDuff, R., Smith, C. and White, A., "Generation of optical phase singularities by computergenerated holograms," Opt. Lett. 17, 221-223 (1992).

[53] Beijersbergen, M. W., Coerwinkel, R. P. C., Kristensen, M.,. Woerdman, J. P, "Helical-wavefront laser beams produced with a spiral phaseplate," Opt. Commun. 112, 321-327 (1994).

[54] Cardano, F., Karimi, E., Slussarenko, S., Marrucci, L., de Lisio, C. and Santamato, E., "Polarization pattern of vector vortex beams generated by q-plates with different topological charges," Appl. Opt. 51, C1-C6 (2012).

[55] Marrucci, L. and Manzo, C. and Paparo, D., "Optical Spin-to-Orbital Angular Momentum Conversion in Inhomogeneous Anisotropic Media,” Phys. Rev. Lett. 96, 163905 (2006).

[56] Cai, X., Wang, J., Strain, M. J., Johnson-Morris, B., Zhu, J., Sorel, M., O’Brien, J. L., Thompson, M. G. and Yu, S., "Integrated Compact Optical Vortex Beam Emitters," Science 338, 363-366 (2012).

[57] Babiker, M., Bennett, C. R., Andrews, D. L. and Dávila-Romero, L. C., "Orbital Angular Momentum Exchange in the Interaction of Twisted Light with Molecules," Phys. Rev. Lett. 89, 143601 (2002).

[58] Williams, M. D., Coles, M. M., Saadi, K., Bradshaw, D. S. and Andrews, D. L., "Optical vortex generation from molecular chromophore arrays," [arXiv:1305.0422] (2013).

[59] Naaman, R. and Waldeck, D. H., "Chiral-induced spin selectivity effect," J. Phys. Chem. Lett. 3, 2178-2187 (2012). 